\title{
CONSTRUÇÃO E AVALIAÇÃO DE UM ELETRODO TUBULAR SENSÍVEL AO ÍON HIDROGÊNIO COMO DETECTOR EM SISTEMAS DE ANÁLISE EM FLUXO
}

\section{Patrícia B. Martelli}

Instituto de Química de São Carlos - Universidade de São Paulo - S. Carlos - SP

Boaventura F. Reis*, Elias A.G. Zagatto

Centro de Energia Nuclear na Agricultura - Universidade de São Paulo - CP 96 - 13.400-970 - Piracicaba - SP

José L.F.C. Lima e Rui A. Lapa

CEQUP/Faculdade de Farmácia - Universidade do Porto - Portugal

\begin{abstract}
CONSTRUCTION AND EVALUATION OF A TUBULAR ION-SELECTIVE ELECTRODE FOR HIDROGEN ION AS A DETECTOR IN FLOW ANALYSIS. The construction of a tubular hydrogen ion-selective potentiometric electrode without inner reference solution, based on the tridodecylamine (TDDA) ionophore, and its evaluation in a flow system are described. TDDA was dissolved in 2-nitrophenyl octyl ether, dispersed in a PVC membrane and applied directly to a conducting support which consisted of an epoxy resin and graphite mixture. The electrode was designed with a tubular geometry to effort facilities to be coupled as part of a flow injection network. The main working characteristics such as response time, linear pH range, selectivity and life time were evaluated and compared with those obtained which a conventionally shaped electrode based on the same sensor. The electrode showed a slope of 51-52 $\mathrm{mV} \mathrm{dec}^{-1}$ within a linear $\mathrm{pH}$ range from 4.0 up to 12.0 .
\end{abstract}

Keywords: tubular pH electrode; flow analysis; tridodecylamine.

\section{INTRODUÇÃO}

$\mathrm{O}$ controle de $\mathrm{pH}$ em laboratórios de rotina, processos industriais, fermentações alcoólicas e em ciências médicas e agronômicas é de suma importância. Para este controle, geralmente, é utilizado o eletrodo de membrana de vidro que é, atualmente o indicador de uso mais generalizado para medições de $\mathrm{pH}$. Outros sensores também tem sido empregados com esta finalidade, e dentre esses destaca-se o eletrodo sensível ao íon hidrogênio, baseado em membrana polimérica. Este tipo de eletrodo foi primeiramente apresentado por Schulthess et alli em 1981, empregando tridodecilamina incorporada em uma matriz de cloreto de polivinila $(\mathrm{PVC})^{1}$. Este eletrodo de membrana polimérica apresentava como vantagens fácil construção, boa estabilidade das medidas, e tem sido utilizado em vários tipos de aplicações ${ }^{2,3}$.

Os eletrodos íon-seletivos (ISEs) de boas características de funcionamento apresentam resposta rápida, boa estabilidade de potenciais ao longo do tempo, baixo custo e além disto, respondem em uma ampla faixa de concentrações. Esta é uma grande vantagem em comparação com outras técnicas de detecção, tais como espectrofotometria, espectrometria de emissão/absorção atômica com chama, polarografia, onde a faixa de resposta dos instrumentos é muito menor. Em consequência, para uma mesma espécie, permite a implementação de sistemas de fluxo mais simples. Adicionalmente, os eletrodos íonseletivos não são afetados pela cor ou turvação das amostras, normalmente não necessitam da adição de um reagente específico, são na maioria dos casos suficientemente seletivos, e fáceis de operar, podendo ser facilmente construídos no laboratório. Estas características tornam a sua aplicação muito abrangente, conforme observa-se na literatura especializada.

Em vista destas características os ISEs têm um grande potencial para serem utilizados em sistemas automatizados de análises, envolvendo determinações multiparamétricas, considerando que as medidas são efetuadas de forma não destrutiva.

Dentre os procedimentos de automação, a análise por injeção em fluxo (FIA) tem sido muito utilizada, pois associa versatilidade, simplicidade e baixo custo das montagens ${ }^{4}$. A detecção potenciométrica empregando ISE em sistemas FIA, foi proposta no sétimo artigo da série publicada por Ruzicka e colaboradores 5 . Neste trabalho, o eletrodo indicador e o de referência foram montados em cascata, para possibilitar que a solução escoasse sobre a superfície sensível do eletrodo indicador. Posteriormente, foram propostos outros arranjos, dentre eles a montagem com jato impingente (wall-jet) que é uma das mais citadas 6 . Enquanto na montagem proposta por Ruzicka e colaboradores ${ }^{5}$, a solução escorria sobre a superfície sensível do eletrodo de trabalho, na montagem com jato impingente a solução era bombeada contra a superfície da membrana sensora.

Nos primeiros trabalhos empregando eletrodos em sistemas FIA, as aplicações foram implementadas com eletrodos convencionais ${ }^{5,7,8}$, o que dificultava o acoplamento dos mesmos nos sistemas, e originava montagens pouco robustas. Visando superar estas dificuldades e obter um melhor aproveitamento das potencialidades do processo FIA, foram desenvolvidos eletrodos com geometrias mais apropriadas para o acoplamento aos módulos de análises ${ }^{6,9}$. Dentre as geometrias propostas, a tubular permite a construção do eletrodo indicador com diâmetro igual ao do percurso analítico, facilitando o acoplamento direto ${ }^{6,10-12}$. Inicialmente, os mesmos foram desenvolvidos com solução de referência interna ${ }^{13,14}$, e posteriormente sem esta solução ${ }^{11}$ ou com contato metálico direto sobre membranas cristalinas homogêneas ${ }^{15}$.

Os eletrodos de geometria tubular podem ser construídos com o diâmetro interno igual ao do percurso analítico, o que evita a diminuição do sinal analítico por dispersão no detector e não altera as características hidrodinâmicas do fluxo. Esta última característica facilita a montagem dos mesmos 
em série no percurso analítico para viabilizar as determinações multiparamétricas ${ }^{16,17}$.

Considerando-se estas características e a utilidade do controle de $\mathrm{pH}$ para estudo de reações químicas em sistemas de análise em fluxo, e a ausência de detectores tubulares com as referidas características sensíveis ao íon $\mathrm{H}_{3} \mathrm{O}^{+}$, o presente trabalho tem como objetivo principal, a construção e avaliação de um eletrodo tubular sensível a $\mathrm{H}_{3} \mathrm{O}^{+}$, sem solução de referência interna, e com resistência mecânica que possibilite de forma robusta a sua fixação ao módulo de análise.

\section{PARTE EXPERIMENTAL}

\subsection{Instrumentos e acessórios}

O módulo de análise era constituído de uma bomba peristáltica Ismatec modelo IPC-8, equipada com tubos de bombeamento de tygon de diferentes diâmetros internos; um injetorcomutador automático ${ }^{18}$; um potenciômetro CRISON, modelo 2002, equipado com um eletrodo de referência de $\mathrm{Ag} / \mathrm{AgCl}$ com dupla junção da ORION, modelo 900029, com uma solução de $\mathrm{KNO}_{3} 10 \%(\mathrm{~m} / \mathrm{v})$ no compartimento externo; um eletrodo tubular sensível ao íon $\mathrm{H}_{3} \mathrm{O}^{+}$; suportes de acrílico para adaptação dos eletrodos ${ }^{19}$; um registrador potenciométrico Radiometer, modelo REC 61; tubos de polietileno de $0,8 \mathrm{~mm}$ de diâmetro interno para construção de reatores e alças de amostragem; válvulas solenóides de 3 vias (NResearch 161T031); um microcomputador IBM 486 equipado com uma interface PCL-711S (American Advantech Corp.) e uma interface eletrônica para acionamento das válvulas solenóides. A comutação do injetor-comutador e o acionamento das válvulas solenóides eram efetuadas pelo microcomputador, empregando um "software" escrito em Quick BASIC $4.5^{20}$.

\subsection{Soluções}

Água destilada e desionizada foi utilizada no preparo das soluções. As soluções de referência empregadas eram preparadas a partir do tampão universal descrito por Perrin e Dempsey $^{21}$ : tetraborato de sódio $5,7 \mathrm{mmol} \mathrm{L}^{-1}$; fosfato dissódico monoácido $20 \mathrm{mmol} \mathrm{L}^{-1}$ e citrato trissódico $13 \mathrm{mmol}$ $\mathrm{L}^{-1}$. Foram feitas soluções para $\mathrm{pH} 3,0 ; 4,0 ; \ldots ; 12,0$, ajustando-se com ácido clorídrico ou hidróxido de sódio. Com esta composição, a intensidade iônica das soluções era de aproximadamente $0,1 \mathrm{~mol} \mathrm{~L}^{-1}$. Diariamente, antes de iniciar os experimentos, determinava-se o $\mathrm{pH}$ destas soluções empregando-se eletrodo de vidro e um potenciômetro com resolução de 0,01 unidades de $\mathrm{pH}$. Previamente o potenciômetro era calibrado com soluções tampão da Merck (pH 4,0 e 7,0).

$\mathrm{Na}$ avaliação dos eletrodos tubulares a solução transportadora utilizada era obtida diluindo-se 100 vezes a solução tampão e ajustando-se o $\mathrm{pH}$ para 3,5.

Para se avaliar o limite inferior de resposta linear do eletrodo, foi utilizada uma solução transportadora $\mathrm{NaOH} 0,01 \mathrm{~mol}$ $\mathrm{L}^{-1}$ e intensidade iônica ajustada com uma solução de $\mathrm{NaCl} 0,1$ mol L ${ }^{-1}$. Neste estudo, as soluções de referência foram preparadas adicionando-se uma solução de $\mathrm{HCl}$ na solução transportadora até se obter diferentes valores de $\mathrm{pH}$. Para cada valor de $\mathrm{pH}$, era registrada a leitura do sinal transiente referente à diferença do potencial.

As soluções dos cátions e ânions interferentes em potencial, $\mathrm{Na}^{+}, \mathrm{K}^{+}, \mathrm{Li}^{+}, \mathrm{NH}_{4}^{+}, \mathrm{Mg}^{2+}, \mathrm{Ca}^{2+}$ e $\mathrm{Cl}^{-}, \mathrm{F}^{-}, \mathrm{Br}^{-}, \mathrm{NO}_{3}^{-}, \mathrm{SO}_{4}{ }^{2-}$, $\mathrm{CH}_{3} \mathrm{COO}^{-}\left(0,01 ; 0,1 ; 0,5 ; 1,0 \mathrm{~mol} \mathrm{~L}^{-1}\right)$, foram preparadas dissolvendo-se os respectivos sais de cloreto e sódio em água. Estas soluções foram empregadas na avaliação dos eletrodos de geometria tubular e convencional.

\subsection{Construção dos ISEs de configuração convencional e tubular}

\subsubsection{Preparação da membrana sensora}

A membrana sensora foi preparada seguindo as recomendações sugeridas por Oesch et alli ${ }^{22}$ e tinha a seguinte composição: Tridodecilamina (TDDA), (FLUKA) 0,02 g; tetraquis-(pclorofenil) borato de potássio (KTpCIPB) $0,14 \mathrm{~g}$; o-nitrofenil octil éter (o-NPOE) 1,4 g; e PVC 0,6 g. TDDA e KTpCIPB foram dissolvidos em o-NPOE e, em seguida, adicionaram-se PVC e $5 \mathrm{~mL}$ de tetrahidrofurano (THF). KTpCIPB foi usado como aditivo visando a diminuição da resistência das membranas e a extensão das interferências aniônicas.

\subsubsection{Preparação do suporte condutor}

O suporte condutor onde as membranas eram aplicadas diretamente, foi preparado misturando-se $0,14 \mathrm{~g}$ de resina epóxi à base de bisfenol (Araldite, Ciba-Geigy S.A), 0,24 g de grafite (granulometria $50 \mu \mathrm{m}$, Merck) e $0,06 \mathrm{~g}$ de endurecedor HR (álcool benzil isoforonediamina, Ciba Geigy S.A). Esta quantidade era suficiente para se preparar dois ou três eletrodos quer de configuração convencional ou tubular.

\subsubsection{Construção dos ISEs de configuração convencional}

O corpo do eletrodo era constituído por um tubo de acrílico (diâmetros: interno $8 \mathrm{~mm}$; externo $10 \mathrm{~mm}$; comprimento 150 $\mathrm{mm}$ ) e um anel também de acrílico (diâmetros: interno $6 \mathrm{~mm}$; externo $8 \mathrm{~mm}$ ) colado no interior do mesmo a $5 \mathrm{~mm}$ de uma das extremidades, no qual foi fixado o contato elétrico. Para isso, empregou-se um disco de cobre com diâmetro de $7 \mathrm{~mm}$, soldado a uma das extremidades do condutor central de um cabo coaxial. Este cabo foi inserido axialmente no tubo de acrílico de modo que o disco de cobre ficasse encostado no anel de acrílico. O espaço entre o disco e a extremidade do tubo de acrílico foi preenchido com a mistura grafite e epóxi cuja preparação foi referida anteriormente. O eletrodo foi colocado em uma estufa a $70^{\circ} \mathrm{C}$ durante um período de 24 horas para o endurecimento da mistura condutora.

A cavidade para aplicação da membrana sensora foi produzida, removendo-se da superfície externa do grafite-epóxi uma camada de aproximadamente $0,5 \mathrm{~mm}$ de espessura. $\mathrm{O}$ corpo do eletrodo foi colocado na posição vertical com a cavidade para cima (Fig. 1) ${ }^{23}$, depositando-se na mesma a solução da membrana sensora, gota a gota, empregando-se uma pipeta de Pasteur. A evaporação do solvente (THF) produziu uma película de PVC sobre a pasta grafite-epóxi. Após evaporação do THF, esta operação era repetida várias vezes, até se preencher completamente a cavidade. $\mathrm{O}$ eletrodo foi deixado à temperatura ambiente durante 24 horas, para secagem completa da membrana.

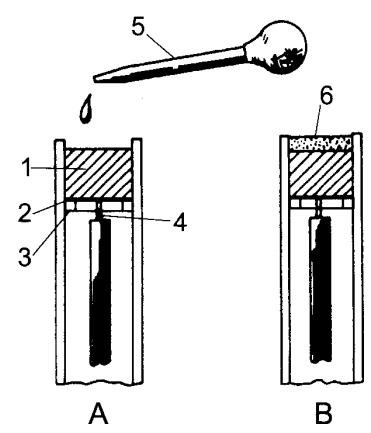

Figura 1. Vista em corte do eletrodo convencional. $A=$ corpo do eletrodo, B=eletrodo com membrana de PVC. $1=$ suporte de resina condutora, $2=$ disco de cobre, $3=$ anel de acrílico, $4=$ condutor elétrico (cabo coaxial), $5=$ solução do sensor e PVC em THF, $6=$ membrana de PVC após a evaporação do solvente. 


\subsubsection{Construção dos ISEs de configuração tubular}

$\mathrm{Na}$ construção do eletrodo tubular (Fig. 2) foi empregado um cilindro de acrílico oco, com as seguinte dimensões: diâmetro interno de $8 \mathrm{~mm}$, externo $10 \mathrm{~mm}$; e comprimento $7 \mathrm{~mm}$ (volume $\cong 4 \mu \mathrm{L}$ ). Através do orifício lateral (A) foi inserido o condutor central de um cabo coaxial (B). Neste condutor, foi soldada uma placa de cobre de $6 \times 2 \mathrm{~mm}$, a qual foi ajustada na parede interna do cilindro de acrílico. O mesmo foi preenchido com a pasta grafite-epóxi, mantendo-se o cabo coaxial encostado na parede do cilindro (C), para se evitar contato entre o material condutor do cabo e a membrana sensora. Após um período de 24 horas a $70{ }^{\circ} \mathrm{C}$ para secagem do suporte condutor, os dois lados do cilindro foram lixados sobre uma superfície plana e, em seguida, recobertos com uma camada de cola Araldite não condutora para se evitar o aparecimento de potenciais mistos causados pelo escoamento das soluções por capilaridade. Após a secagem da Araldite, fez-se um furo de 1 $\mathrm{mm}$ de diâmetro no centro do cilindro no sentido axial (D). Neste canal, foi gotejada a solução da membrana sensora, empregando-se uma pipeta de Pasteur. Para garantir o recobrimento de toda a superfície do canal, esta operação foi repetida 10 vezes, esperando-se um intervalo de 30 minutos entre cada aplicação, para dar tempo à evaporação do solvente. Após o recobrimento com a membrana sensora o diâmetro interno do canal era de aproximadamente $0,8 \mathrm{~mm}$.

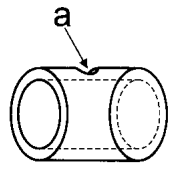

A
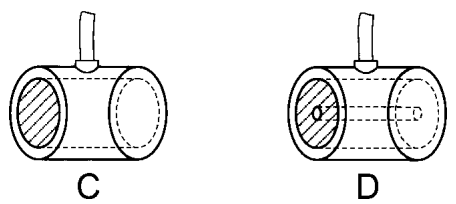

Figura 2. Etapas de preparação do eletrodo tubular. A=molde de acrílico, diâmetros interno e externo 8 e $10 \mathrm{~mm}$, comprimento $7 \mathrm{~mm}$, $a=$ furo de $1 \mathrm{~mm}$ de diâmetro; $B=$ adaptação do condutor central do cabo coaxial com a placa de cobre soldada; $C=$ molde preenchido com a pasta grafite-epóxi; $D=v i s t a$ do eletrodo mostrando o canal onde foi depositada a membrana de PVC

$\mathrm{Na}$ aplicação do sensor, evitou-se a inundação do canal, pois com a evaporação do solvente poderia ocorrer obstrução do mesmo. Após completar estas operações, deixou-se o eletrodo em repouso, em temperatura ambiente durante 24 horas, para garantir total evaporação do solvente. É inevitável que durante a aplicação da solução da membrana sensora, um excesso escorra sobre a superfície externa do grafite-epóxi. Após a secagem este excesso era cuidadosamente removido. Finalizadas estas etapas, efetuaram-se as conexões elétricas e a montagem do eletrodo, conforme mostra a figura $3^{24}$.

O eletrodo foi encaixado em um suporte de acrílico construído para esta finalidade, permitindo que, ao ser acoplado ao módulo de análise, a solução transportadora fluísse através do canal. Na figura $4^{19}$ são mostrados os detalhes da construção e da montagem.

\subsubsection{Condicionamento dos eletrodos íon-seletivos}

Após a secagem da membrana sensora dos ISEs tubulares esta era condicionada, bombeando-se uma solução de $\mathrm{HCl}$ $10^{-3} \mathrm{~mol} \mathrm{~L}^{-1}$ durante 2 horas. Quando o eletrodo não estava

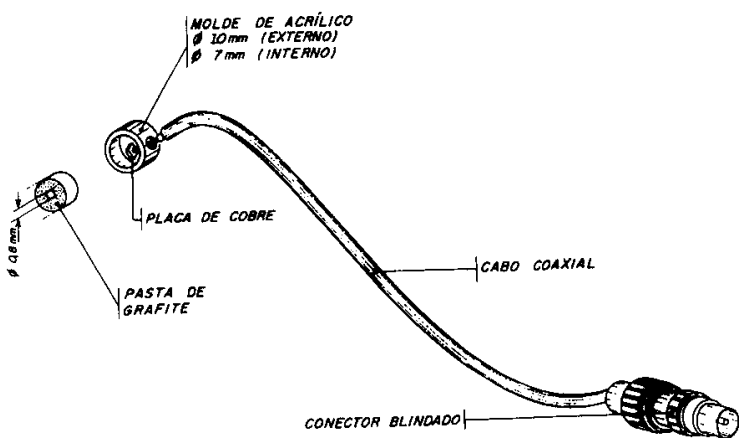

Figura 3. Detalhes do eletrodo tubular.

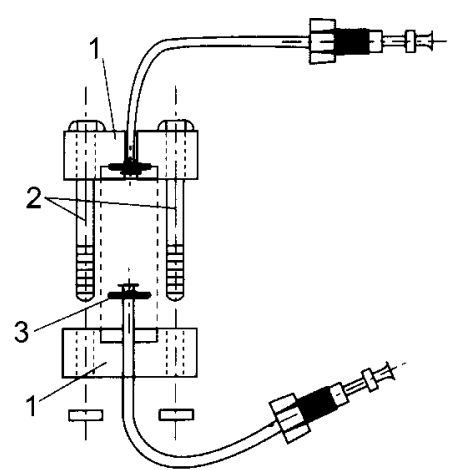

A

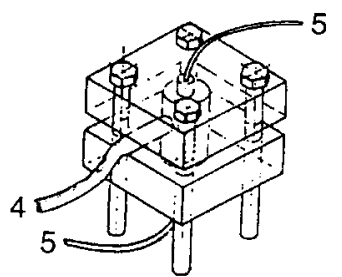

$\mathrm{B}$
Figura 4. Detalhes da montagem final do eletrodo. $A=v i s t a$ do suporte em corte, $B=v i s t a$ do eletrodo tubular adaptado no suporte. $1=s u$ portes de acrílico; 2=parafusos; 3=anéis de borracha de silicone; $4=$ cabo coaxial; 5=tubos de polietileno (entrada e saída de fluído).

sendo utilizado, o canal era preenchido com água destilada. Para evitar a evaporação, fechavam-se as extremidades das luvas de conexão. Ao se iniciar cada período de trabalho, o eletrodo era recondicionado, bombeando-se através dele uma solução de $\mathrm{HCl} 10^{-3} \mathrm{~mol} \mathrm{~L}^{-1}$ durante 15 minutos. Os eletrodos de configuração convencional eram condicionados ou mantidos entre utilizações imersos em soluções de composição semelhante às referidas para os ISEs de configuração tubular.

\subsection{Avaliação dos eletrodos íon-seletivos}

\subsubsection{ISE de configuração convencional}

As características gerais de funcionamento dos ISEs de configuração convencional foram avaliadas visando estabelecer, ao longo do trabalho, comparações sistemáticas com os ISEs de configuração tubular. Nomeadamente, foram determinadas a faixa de resposta linear, a inclinação das curvas de calibração, a repetibilidade dos potenciais, o tempo de resposta e o tempo de vida. Para isto foram efetuadas calibrações repetidas com soluções padrão na faixa de $\mathrm{pH}$ de 4,0 a 12,0 . Estes experimentos foram realizados de maneira convencional, isto é, colocando-se a solução em um copo sob agitação contínua, e medindo-se a diferença de potencial correspondente.

\subsubsection{ISE de configuração tubular}

A avaliação do eletrodo de configuração tubular foi realizada empregando-se o módulo de análise cujo diagrama de fluxos é mostrado na figura 5. O injetor está na posição de amostragem, a solução de referência $(\mathrm{S})$ está sendo aspirada através da válvula (V) para preencher a alça de amostragem (L), que 
define o volume a ser injetado e o excesso é descartado (D). Deslocando-se o injetor para outra posição de repouso, o conteúdo da alça de amostragem é deslocado pela solução transportadora (C) em direção ao ISE tubular. O sinal transiente correspondente é detectado e traçado em função do tempo pelo registrador potenciométrico.

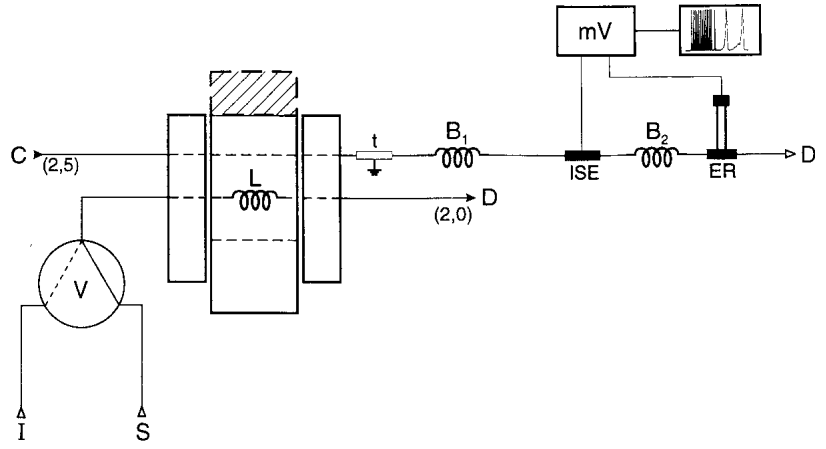

$\mathrm{T}_{\mathrm{V}}$

Figura 5. Diafragma de fluxos do sistema para avaliação do eletrodo tubular. As três barras retangulares representam o injetor visto de cima, a área hachurada indica o deslocamento da parte móvel do injetor para a posição de injeção e as linhas tracejadas indicam canais internos. $S=$ solução de referência; $I=$ solução do ion interferente; $C=$ solução transportadora $(\mathrm{pH}=3,5) ; V=$ válvula solenóide; ; $L=$ alça de amostragem (40 $\mathrm{cm}) ; t=$ tubo de aço-inox, $10 \mathrm{~mm}$ de comprimento e $1 \mathrm{~mm}$ d.i.; $D=$ descarte; $I S E=$ eletrodo tubular (indicador); $E R=$ eletrodo de referência; $B_{I}=$ linha de conexão do injetor ao eletrodo indicador $(4 \mathrm{~cm}) ; B_{2}=$ linha de conexão do eletrodo indicador ao de referência $(5 \mathrm{~cm}) ; m V=$ potenciometro; $T_{v}=$ diagrama de tempo de acionamento da válvula: a linha em nível alto significa que a válvula está acionada. A linha contínua através do símbolo da válvula indica a passagem da solução com a válvula desligada e a linha tracejada indica a passagem da solução quando a válvula é acionada. Os números entre parênteses correspondem às vazões de bombeamento em $\mathrm{mL} \mathrm{min}^{-1}$.

Usando esta montagem foram executados experimentos para se avaliar o desempenho do eletrodo tubular no que se refere à faixa de resposta linear, à inclinação das curvas de calibração e à estabilidade dos potenciais ao longo do tempo. Além destes parâmetros, foi determinado o tempo de resposta para as soluções de referência de pH 5,0 e 11,0 com uma vazão da solução transportadora de $2,5 \mathrm{~mL} \mathrm{~min}^{-1}$.

O tempo de resposta de um detector potenciométrico é um parâmetro importante, principalmente quando o mesmo é utilizado em sistemas FIA. Para se determinar o tempo de resposta dos ISEs de configuração tubular, foi projetado o módulo de análise cujo diagrama de fluxos é mostrado na figura 6.

$\mathrm{Na}$ posição indicada nesta figura, as válvulas estão desligadas, e somente a solução transportadora está sendo bombeada. Nestas condições, tem-se o registro correspondente ao $\mathrm{pH}$ da solução transportadora. Acionando-se as válvulas $\mathrm{V}_{1} \mathrm{e} \mathrm{V}_{2}$ de acordo com o diagrama de tempo, a solução transportadora (C) é desviada para recuperação $\left(\operatorname{Re}_{C}\right)$ e em seu lugar, é bombeada a solução de referência (S). Desligando-se as duas válvulas, interrompe-se o fluxo da solução de referência, restabelecendo-se o fluxo da solução transportadora. Para se minimizar os efeitos da pulsação da bomba peristáltica na precisão das medidas, acoplaram-se amortecedores de pulsos (Am) entre a bomba peristáltica e as válvulas ${ }^{25}$. A bobina $\left(\mathrm{B}_{0}\right)$ aumentava a impedância hidrodinâmica entre o amortecedor de pulsos e o percurso analítico, melhorando a eficiência do amortecedor.

Para se avaliar o tempo de resposta do eletrodo, foram executados experimentos programando-se intervalos de tem-

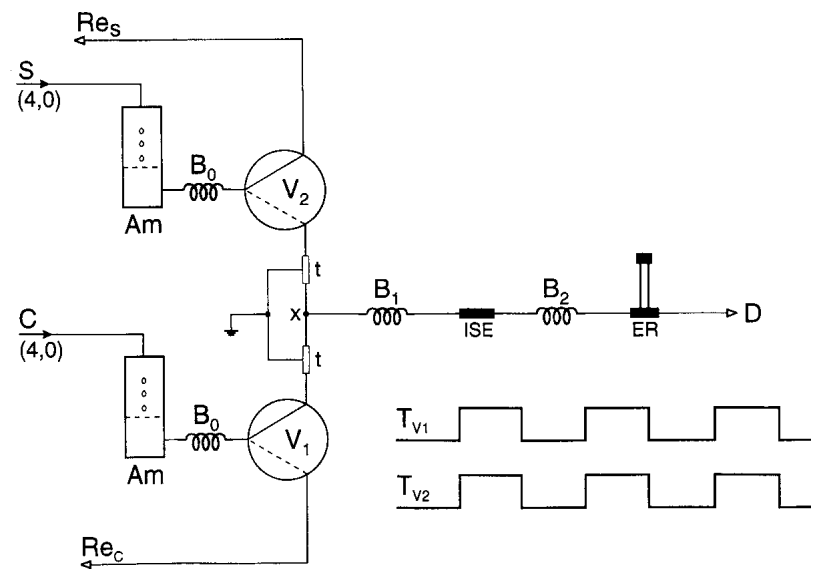

Figura 6. Diagrama de fluxos do sistema de análise para verificar o tempo de resposta do eletrodo. $S=$ solução de referência, $R_{e s}=$ recuperação da solução de referência, C=solução transportadora $(p H=3,5)$, $R_{e c}=$ recuperação da solução transportadora, Am=amortecedor de pulsos, $V_{1}$ e $V_{2}=$ válvulas solenóide de 3 vias, $B_{0}=150 \mathrm{~mm}, B_{1}=1 \mathrm{~cm}$, $B_{2}=5 \mathrm{~cm}$. $T_{V 1}$ e $T_{V 2}=$ correspondem aos tempos de acionamento das válvulas $V_{1}$ e $V_{2}$, respectivamente. Os demais parâmetros e símbolos são os mesmos da figura 5.

po de 0,$25 ; 0,50 ; 1,00 ; 2,00 ; 4,00$ e $6,00 \mathrm{~s}$ para acionamento das válvulas. Este ciclo foi repetido várias vezes, usando-se soluções de referência de pH 5,0 e 11,0. Os sinais gerados foram traçados com registrador potenciométrico em função do tempo.

Os testes que visavam a avaliação da extensão das interferências foram executados, operando-se o módulo de análise cujo diagrama de fluxo é mostrado na figura 5, sendo que a válvula (V) é acionada para promover a adição da solução do íon interferente às soluções de referência. Quando a válvula (V) é acionada, a solução interferente (I) é aspirada no lugar da solução de referência (S). O programa de controle foi desenvolvido para acionar a válvula seguindo o diagrama de tempo (Tv). Assim a alça de amostragem é preenchida com uma sequência de pequenas alíquotas da solução do interferente separadas por alíquotas da solução de referência.

A permanência da válvula na posição de aspiração de cada uma das soluções foi fixada em $0,2 \mathrm{~s}$. Em função do comprimento da alça de amostragem, foram programados 25 pares de alíquotas das duas soluções para garantir o preenchimento completo da mesma. Deslocando-se o injetor para a outra posição de repouso, o conteúdo da alça de amostragem é deslocado pela solução transportadora (C) em direção ao ISE tubular. O sinal transiente correspondente é detectado e analogamente registrado. Assim, em cada ciclo, a alça de amostragem era carregada com a mistura das soluções de referência e do íon interferente na proporção de 1:1. Em cada conjunto de experimentos envolvendo as soluções de um íon interferente, o sinal somente do íon principal $\left(\mathrm{H}_{3} \mathrm{O}^{+}\right)$era obtido, substituindo-se a solução do íon interferente pela solução transportadora. A mesma sequência de experimentos foi efetuada para todas as soluções descritas no item 2.2.

\section{RESULTADOS E DISCUSSÃO}

As características gerais de funcionamento dos ISEs de configuração convencional foram avaliadas por procedimentos tradicionais, efetuando-se as medidas por processos discretos ("batch"). No caso dos ISEs tubulares, as mesmas características foram obtidas, usando-se uma solução transportadora de baixa capacidade tampão $\left(\approx 10^{-5} \mathrm{~mol} \mathrm{~L}^{-1}, \mathrm{pH}=3,5\right)$, evitandose que a mesma alterasse a acidez da zona da amostra, conforme sugerido por outros autores ${ }^{9,26}$. Embora nestas condições a intensidade iônica da solução transportadora não seja elevada, 
o contato permanente da membrana sensora com uma solução em que o íon $\mathrm{H}_{3} \mathrm{O}^{+}$estava sempre presente, fornecia uma linha base estável ao longo do tempo. Esta característica é muito importante para garantir a qualidade dos resultados obtidos.

$\mathrm{O}$ volume da solução da amostra utilizado nestes experimentos era de $200 \mu \mathrm{L}$, enquanto o volume do percurso analítico era de $25 \mu \mathrm{L}$, portanto o suficiente para permitir que a leitura fosse efetuada praticamente em condição de estado estacionário. Com esta configuração evitava-se que a diferença da intensidade iônica da solução transportadora afetasse a resposta do eletrodo. Convém salientar que a leitura correspondente à solução transportadora não foi considerada na análise dos resultados.

A resposta do ISE de configuração tubular em função da concentração do íon principal, expressa em valores de $\mathrm{pH}$, é mostrada na figura 7 , onde se observa uma resposta linear no intervalo de $\mathrm{pH}$ de 4,0 a 12,0 e inclinação de $51,6 \mathrm{mV}$ por década de concentração. Estas características se mantinham sem alterações significativas $(<5,0 \mathrm{mV})$ pelo menos durante um dia de trabalho. Este comportamento dispensa, em trabalho de rotina, o recurso das calibrações sistemáticas.

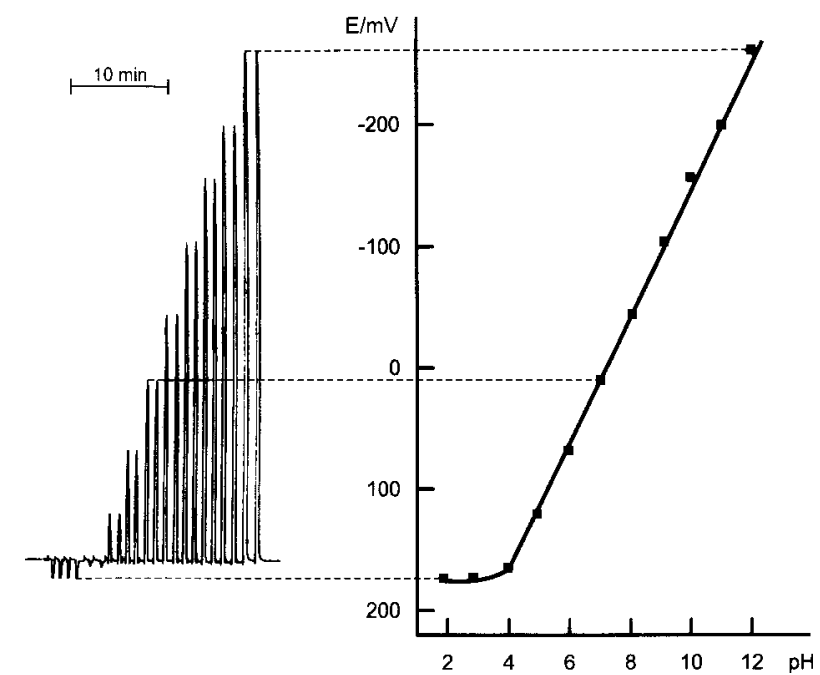

Figura 7. Registro típico e curva analítica. Vazão da solução transportadora $=2,5 \mathrm{~mL} \mathrm{~min}^{-1}$ ( $\left.\mathrm{pH}=3,5\right)$; alça de amostragem (40cm). Neste experimento a solução do ion intereferente (figura 5) foi substituída pela solução transportadora.

O desempenho dos ISEs de configuração tubular e convencional ao longo do tempo foi averiguado traçando-se sucessivas curvas analíticas (com intervalos de $30 \mathrm{~min}$ ) durante um período de 4 horas. Os resultados obtidos evidenciaram um amplo intervalo de resposta linear $(\mathrm{E}=396,64-51,64 \mathrm{pH}$, $r=0,9998$ ), uma boa repetibilidade ao longo do tempo (desvio padrão relativo $<4 \%$ ). Conforme observado na tabela 1 , os resultados não diferem significativamente dos apresentados pelos eletrodos de configuração convencional, avaliados por métodos descontínuos.

Tabela 1. Características gerais dos eletrodos

\begin{tabular}{lcc}
\hline Parâmetros estudados & $\begin{array}{c}\text { Eletrodo } \\
\text { convencional }\end{array}$ & $\begin{array}{c}\text { Eletrodo } \\
\text { tubular }\end{array}$ \\
\hline Intervalo de resposta linear $(\mathrm{pH})$ & $3,5-12$ & $3,5-12$ \\
Menor valor de $\mathrm{pH}$ & $3,5 \pm 0,7$ & $3,5 \pm 0,7$ \\
Coeficiente angular* $\left(\mathrm{mV} \mathrm{dec}^{-1}\right)$ & $53,7 \pm 0,3$ & $51,6 \pm 0,7$ \\
Tempo de resposta $(\mathrm{s})$ & - & $<3$ \\
\hline
\end{tabular}

* Valores médios calculados considerando-se 8 medidas consecutivas.
Considerando-se que em sistemas FIA a detecção é feita com a amostra em movimento em relação ao detector, impõe-se que o transdutor apresente uma resposta rápida, para que as medidas possam realizar-se em condições próximas de tempo real, nomeadamente quando se procuram condições de máxima sensibilidade. Assim, efetuaram-se experimentos para avaliar o tempo de resposta do eletrodo tubular (Fig. 8). Este parâmetro foi obtido, considerando-se o intervalo de tempo necessário para o valor do potencial alcançar $95 \%$ do valor correspondente ao estado estacionário. A motivação para adotar este valor, deve-se ao fato de este critério ter sido empregado na primeira publicação sobre ISE com membrana plástica e sem solução de referência interna, onde este parâmetro foi avaliado ${ }^{11}$.

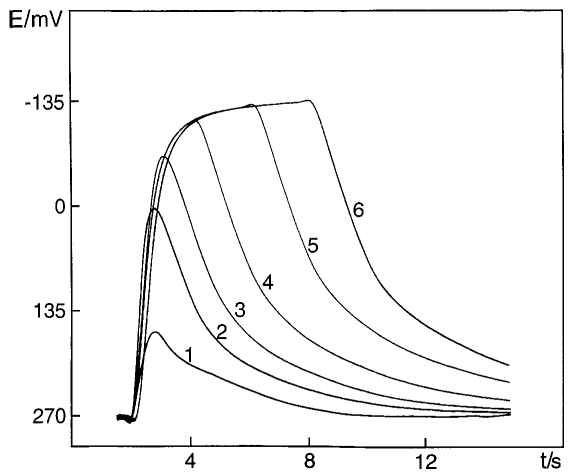

Figura 8. Efeito do volume injetado. As curvas 1, 2, .., 6 correspondem aos intervalos de tempos de 0,25;0,50;1,00;2,00;4,00 e 6,00s programados para o acionamento das válvulas (figura 6). Solução de referência $\mathrm{pH}=11,0$ e solução transportadora $\mathrm{pH}=3,5$.

Visando obter resultados mais independentes possíveis da dispersão da amostra na solução transportadora, a distância entre o ponto de inserção da solução da amostra e o eletrodo indicador foi fixada em $10 \mathrm{~mm}$ (Fig. 6). Com este arranjo, a solução da amostra ao atingir o detector, apresentava uma concentração muito próxima à do instante da injeção. A vazão da solução transportadora foi estabelecida em $66,7 \mu \mathrm{L} \mathrm{s}^{-1}$, apresentando como resultado um tempo de trânsito da amostra, entre o ponto de inserção e o eletrodo indicador, de apenas $75 \mathrm{~ms}$.

A partir da figura 8, tomando-se como referência o início dos traçados das curvas, deduz-se que o intervalo de tempo necessário para o sinal alcançar $95 \%$ do valor do estado estacionário, quando se intercala uma solução de $\mathrm{pH}=11,0$ foi de aproximadamente 2,8 s. As curvas 1,2 e 3 não foram consideradas neste cálculo, pois conforme mostra a figura, as respectivas leituras não alcançavam um estado estacionário. Na dedução deste tempo de resposta, não foi considerado o tempo de trânsito da solução de referência, porque o valor encontrado é muito maior (37 vezes) que o tempo de trânsito. Experimentos análogos foram executados usando-se uma solução com pH 5,0 e a partir dos resultados, encontrou-se um tempo de resposta em torno de $2 \mathrm{~s}$. $\mathrm{O}$ valor limite de $2,8 \mathrm{~s}$ foi considerado durante o projeto do módulo de análise, pois o intervalo de tempo para o trânsito da solução de referência através do eletrodo tubular não pode ser menor que este valor. Esta característica impõe um limite no menor volume de amostra que deve ser injetado.

A maior velocidade de resposta obtida quando se intercala a solução de $\mathrm{pH}=5,0$ relativamente à intercalação de soluções de $\mathrm{pH}=11,0$ é um resultado esperado, quando se utilizam detectores potenciométricos ${ }^{27}$.

Para se efetuar a avaliação da extensão das interferências causadas por outras espécies iônicas, o sistema FIA (Fig. 5) foi projetado para efetuar, durante a amostragem, a mistura da solução principal com as soluções dos íons interferentes. Foi empregado o processo de amostragem binária ${ }^{28}$, sendo a alça de amostragem 
preenchida com a mistura das duas soluções na razão de $50 \%$. A vazão de amostragem foi fixada em $33,3 \mu \mathrm{L} \mathrm{s}^{-1}$ e o intervalo de tempo de acionamento repetitivo da válvula $\mathrm{V}$ (ligada/desligada) foi fixado em 0,2 s. Deste modo, a alça de amostragem era preenchida com uma sequência de pequenas alíquotas de amostra $(6,7 \mu \mathrm{L})$, separadas entre si por alíquotas de igual volume da solução do íon interferente. Em cada ciclo de análise, foi programada a amostragem de 25 pares de alíquotas, o que correspondia a um volume de $335 \mu \mathrm{L}$. O volume da alça de amostragem era de $200 \mu \mathrm{L}$, e quando o injetor retornava para a posição de amostragem, estava preenchida com a solução transportadora. Aspirandose um volume de $335 \mu \mathrm{L}$, evitava-se a contribuição da solução transportadora para formação de gradientes de $\mathrm{pH}$, pois os primeiros $135 \mu \mathrm{L}$ eram descartados.

O comprimento da alça de amostragem era $400 \mathrm{~mm}$, e cada alíquota das duas soluções ocupava um espaço de $13,4 \mathrm{~mm}$ da mesma (30 vezes menor). Nestas condições uma boa coalescência entre as duas soluções era alcançada durante a etapa de amostragem, minimizando-se a formação de gradientes de $\mathrm{pH}$. Durante o deslocamento da zona de amostra para o detector, ocorria mútua dispersão a partir das duas interfaces de contato entre a solução transportadora e da amostra. Em consequência, era gerado um gradiente de $\mathrm{pH}$ positivo e outro negativo. Isto poderia dificultar a determinação do tempo de resposta do eletrodo. Em vista disto, para se obter uma região de leitura constante em função do tempo (condição de "volume infinito"), fixou-se um percurso analítico (B1, Fig. 5) oito vezes menor que a alça de amostragem.

Com relação à extensão das interferências, verifica-se na tabela 2, que o íon amônio apresenta interferência mais acentuada, diminuindo a faixa de resposta linear na zona alcalina. Presumivelmente, isto ocorre devido à conversão do amônio para amônia, consequentemente alterando o equilíbrio entre os íons $\mathrm{H}_{3} \mathrm{O}^{+}$e $\mathrm{OH}^{-}$.

Tabela 2. Efeito dos interferentes na faixa de resposta linear em função do $\mathrm{pH}$

\begin{tabular}{ccc}
\hline Interferentes & Concentração/mol L $\mathrm{m}^{-1}$ \\
\hline $\mathrm{NH}_{4}^{+}$ & 0,01 & $4,0-9,0$ \\
$\mathrm{~K}^{+}$ & 0,50 & $5,0-12,0$ \\
$\mathrm{Li}^{+}$ & 1,00 & $5,0-12,0$ \\
$\mathrm{Na}^{+}$ & 0,50 & $4,5-12,0$ \\
$\mathrm{Ca}^{2+}$ & 0,01 & $4,0-10,0$ \\
$\mathrm{Mg}^{2+}$ & 0,01 & $4,0-9,5$ \\
$\mathrm{Acetato}^{2+}$ & 0,10 & $6,0-12,0$ \\
$\mathrm{NO}_{3}^{-}$ & 0,50 & $6,0-12,0$ \\
$\mathrm{Cl}^{-}$ & 0,50 & $5,0-12,0$ \\
$\mathrm{Br}^{-}$ & 0,10 & $5,0-12,0$ \\
$\mathrm{~F}^{-}$ & 0,10 & $5,5-12,0$ \\
$\mathrm{SO}_{4}{ }^{-}$ & 1,00 & $4,5-12,0$ \\
\hline
\end{tabular}

Quanto aos ânions, maior interferência foi observada para o íon acetato, provavelmente causada pela formação de sistema tampão no percurso analítico. Em $\mathrm{pH}$ mais baixo observou-se que este efeito era bem mais acentuado o que, em princípio, confirma esta suposição.

Dentre os ânions estudados, o eletrodo apresentou faixa de resposta linear mais ampla para o íon sulfato, mesmo quando foi utilizada solução $1,0 \mathrm{~mol} \mathrm{~L}^{-1}$. Este comportamento é semelhante ao citado na literatura para eletrodos de configuração convencional com um sistema sensor de composição semelhante ${ }^{29}$.

Embora não se tenham realizado experimentos sistemáticos no sentido de determinar o tempo de vida médio destes ISEs, pode-se dizer que as unidades desenvolvidas se mantém em boas condições de funcionamento por um período superior a um ano. Durante este tempo mais de 2.000 medidas foram realizadas em épocas distintas. Estes resultados confirmam outros anteriormente obtidos com ISEs de construção semelhante e sensíveis a outras espécies, evidenciando a vantagem dos processos de construção em que não são utilizados eletrodos com soluções de referência interna ${ }^{11}$.

\section{CONCLUSÕES}

Até onde estamos informados, este é primeira unidade de ISE tubular sensível ao íon $\mathrm{H}_{3} \mathrm{O}^{+}$. Os resultados da avaliação indicaram um bom desempenho, nomeadamente o que se refere à velocidade de resposta, faixa de resposta linear e tempo de vida. A geometria tubular, facilita o acoplamento no módulo de análise em fluxo, resultando em montagens com grande estabilidade mecânica e viabilizando as implementações das determinações de uma forma robusta. Além disto apresenta um baixo custo, o que o torna acessível a qualquer laboratório de pesquisa ou de controle.

A disponibilidade de detectores potenciométricos tubulares constitui uma importante ferramenta para estudos de características hidrodinâmicas em sistemas FIA, considerando que o sensor está incorporado no módulo de análise e não origina perturbação no segmento da solução da amostra.

Em termos gerais, as características de funcionamento dos ISEs de configuração tubular, em sistemas FIA de baixa dispersão são semelhantes, ou mesmo melhores, que as dos eletrodos de geometria plana com o mesmo tipo de membrana ${ }^{9,29}$, apresentando como vantagem adicional a facilidade de acoplamento.

O tempo de vida das unidades é elevado quando comparado com os dos eletrodos de construção convencional que incorporam um eletrodo e uma solução de referência interna. Na maioria dos casos referidos na literatura, nunca se mantém as características de funcionamento por períodos superiores a 6 meses ${ }^{16,30}$.

A velocidade de resposta dos ISEs de configuração tubular é perfeitamente adequada à sua incorporação em sistemas FIA, sendo manifestamente superior à dos ISE convencionais, devido ao papel condicionador do fluido transportador. Esta última característica associada ao pequeno volume interno do mesmo $(\approx 4 \mu \mathrm{L})$, tornam-o ideal para acoplamento no percurso analítico em sistemas FIA. O pequeno volume é uma característica fundamental, principalmente, para estudos em que seja necessário além da resposta rápida do sensor, manter inalterada a estrutura da zona da amostra. Por exemplo, em sistemas com relocação do detector ${ }^{31}$, teriam como resultado favorável o aumento da frequência de amostragem.

O conjunto de características referidas permitem prever para o ISE de configuração tubular um amplo leque de aplicações, designadamente como eletrodo indicador em titulações em fluxo contínuo. Neste contexto, deve-se salientar que o sistema proposto fornece informações adicionais relativamente à capacidade tampão e às constantes de equilíbrio envolvidas.

\section{AGRADECIMENTOS}

Os autores agradecem à permuta de investigadores financiada pela Ação Integrada FAPESP/JNICT. Os investigadores brasileiros agradecem à FAPESP, ao $\mathrm{CNPq}$ e à CAPES e a parte portuguesa à JNICT pelo financiamento obtido através do Projeto PBICT/C/QUI/2165/95.

\section{REFERÊNCIAS}

1. Schulthess, P.; Shijo, Y.; Pham, H. V.; Pretsch, E.; Ammann, D.; Simon, W.; Anal. Chim. Acta 1981, 131, 111.

2. Lima, J. L. F. C.; Machado, A. A. S. C.; Quim. Nova 1987, 10, 137.

3. Alonso, A.; Bartroli, J.; Lima, J. L. F. C.; Raurich, J.G.; Quim. Anal. 1991, 10, 335. 
4. Ruzicka, J.; Hansen, E. H.; Anal. Chim. Acta 1975, $78,145$.

5. Ruzicka, J.; Hansen, E. H.; Zagatto, E. A. G.; Anal.Chim. Acta 1977, 88, 1 .

6. Ferreira, I. M. P. L. V. O.; Lima, J. L. F. C.; J. Flow Injection Anal. 1993, 10, 17.

7. Slanina, J.; Lingerak, W. A.; Anal. Chim. Acta 1980, $117,91$.

8. Opdycke, W. N.; Parks, S. J.; Meyerhoff, M. E.; Anal. Chim. Acta 1983, 155, 11.

9. Hauser, P. C; Cardwell, T. J; Cattrall, R. W; Tan, S. S; Hamilton, I. C.; Anal. Chim. Acta 1989, 221, 139.

10. Montenegro, M. C. B. S. M.; Lima, J. L. F. C.; Mattos, I. L.; Oliveira Neto, G.; Gomes Neto, J. A.; Zagatto, E. A. G.; Talanta 1993, 40, 1563.

11. Alegret, S.; Alonso, J.; Bartroli, J.; Paulís, J. M.; Lima, J. L. F. C.; Machado, A.A. S.C.; Anal. Chim. Acta 1984, 164,147

12. Araújo, A. N.; Etxebarria, M. B.; Lima, J. L. F. C.; Montenegro, M. C. B. S. M.; Olmos, R. P.; Anal. Chim. Acta 1994, 293, 35 .

13. Meyerhoff, M. E.; Fraticelli, P. M.; Anal. Lett. 1981, 14,415 .

14. Frend, A. J.; Moody, G. J.; Thomas, J. D. R.; Birch, B. J.; Analyst 1983, 108, 1357.

15. Ferreira, I. M. P. L. V. O.; Lima, J. L. F. C.; Rocha, L. S. M.; Fresenius J. Anal. Chem. 1993, 347, 314.

16. Ferreira, I. M. P. L. V. O.; Lima, J. L. F. C.; Montenegro, M. C. B. S. M.; Olmos, R. P.; Rios, A.; Analyst 1996, 121, 1393.

17. Alonso, J.; Bartroli, J.; Jun, S.; Lima, J. L. F. C.; Montenegro, M. C. B. S. M.; Analyst 1993, 118, 1527.
18. Reis, B. F.; Zagatto, E. A. G.; Martelli, P. B.; Brienza, S. M. B.; Analyst 1993, 118, 719.

19. Alegret, S.; Alonso, J.; Bartroli, J.; Machado, A. A. S. C.; Lima, J. L. F. C.; Paulis, J. M.; Quim. Anal. 1987, 6, 278.

20. Martelli, P. B.; Reis, B. F.; Kronka, E. A. M.; Bergamin Filho, H.; Korn, M.; Zagatto, E. A. G.; Lima, J. L. F. C.; Araújo, A. N.; Anal. Chim. Acta 1995, 308, 397.

21. Perrin, D. D.; Dempsey, B.; Buffers for $\mathrm{pH}$ and Metal Ion Control, Chapman and Hall, London, 1974.

22. Oesch, U.; Brzózka, Z.; Xu, A.; Rusterholz, B.; Suter, G.; Pham, H. V.; Welti, D. H.; Ammann, D.; Pretsch, E.; Simon, W.; Anal. Chem. 1986, 58, 2285.

23. Lima, J. L. F. C.; Machado, A. A. S. C.; Analyst 1986, $111,799$.

24. Gomes Neto, J. A.; Tese de Doutorado, Instituto de Química de São Carlos-USP, 1995.

25. Korn, M.; Gouveia, L. F. B. P.; Oliveira, E.; Reis, B. F.; Anal. Chim. Acta 1995, 313, 177.

26. Hongbo, C.; Hansen, E. H.; Ruzicka, J.; Anal. Chim. Acta 1985, 169, 209.

27. Covington, K. A.; Ion-Selective Electrode Methodology, CRC Press, Inc. Boca Raton, Florida, 1984, v. I, Cap. 3, p. 49.

28. Reis, B. F.; Giné, M. F.; Zagatto, E. A. G.; Lima, J. L. F. C.; Lapa, R. A.; Anal. Chim. Acta 1994, 293, 129.

29. Alegret, S.; Raurich, J. G.; Porcel, C. I.; Fàbregas, E. M.; Martorell, D.; Quim. Anal. 1994, 13, 176.

30. Moody, G. J.; Thomas, J. D. R.; Lima, J. L. F. C.; Machado, A. S. C.; Analyst 1988, 113, 1023.

31. Gomes Neto, J. A.; Nogueira, A. R. A.; Bergamin Filho, H.; Zagatto, E. A. G.; Montenegro, M. C. B. S.; Lima, J. L. F. C.; Anal. Chim. Acta 1994, 285, 293. 